\title{
Statistical models for intensity and phase fluctuation of laser light in a turbulent medium
}

\author{
WANG HongXing, LIU Min*, WANG Qian, ZHANG TieYing \& LIU XiGuo \\ Department of Electronic and Information Engineering, Navy Aeronautical and Astronautical University, Yantai 264001, China
}

Received October 25, 2010; accepted December 31, 2010; published online April 13, 2011

\begin{abstract}
The probability density function of irradiance fluctuations in a turbulent atmosphere remains an open topic of research. It is generally considered that the speckle field has an elliptical-Gaussian speckle distribution, but because of its complexity there is still no exact analytical solution. By introducing the concept of random fluctuation intensity and using an equiprobability density ellipse, exact models of the random fluctuation intensity and its phase are proposed. Through theoretical analysis and numerical simulation, it is concluded that the random fluctuation intensity and its corresponding phase are independent only under the circular-Gaussian approximation.
\end{abstract}

optical propagation, atmospheric turbulence, speckle field, equiprobability density ellipse, random fluctuation intensity, phase, probability density function

Citation: Wang H X, Liu M, Wang Q, et al. Statistical models for intensity and phase fluctuation of laser light in a turbulent medium. Chinese Sci Bull, 2011, 56: 1539-1542, doi: 10.1007/s11434-011-4448-5

The performance of a lasercom system operating in the atmosphere is reduced by irradiance scintillations and phase fluctuations in the received signal due to optical turbulence [1]. Because of the needs of optical communication and atmospheric remote sensing, the probability density function (PDF) of the irradiance scintillations and phase fluctuations is a problem of continuing interest [2,3]. Initially, the results of most theoretical studies based on the circular-Gaussian speckle distribution did not agree well with reported measurements [4-6]. Therefore, Bissonnette and Wizinowich [7] proposed the elliptical-Gaussian speckle distribution and derived the PDF of intensity in strong turbulence through proper approximation in 1979. Fremouw et al. [8] experimentally showed the rationality of the elliptical-Gaussian speckle distribution in describing the statistical characteristics of the turbulent field. However, because of the complexity of the elliptical-Gaussian speckle distribution, all existing models, especially intensity models, are approximations. Researchers have made efforts to derive an exact model $[9,10]$, but have not yet succeeded. Zhang et al.

*Corresponding author (email: liumin05@163.com)
[11] proposed the concept of random fluctuation intensity to express the intensity fluctuations. Assuming independence, they derived models of the random fluctuation intensity and its corresponding phase, and then produced models of the intensity and phase fluctuations. Although they were able to derive models of intensity and phase fluctuations, the approximations and phenomenological modeling concepts employed in deducing the new models limit their applicability. Here, employing the concept of the equiprobability density ellipse, we mathematically deduce exact models of the random fluctuation intensity and its corresponding phase, and conclude that they are exactly independent under the circular-Gaussian approximation. Although it is impossible to derive the models of intensity and phase fluctuations using these exact models, the random fluctuation intensity itself is a useful parameter to describe the intensity fluctuation.

\section{Elliptical-Gaussian speckle field}

\subsection{Born perturbation theory}

Laser light propagating in turbulent media has two 
characters: randomicity of the media, which leads to the use of a statistical method, and weak fluctuation of the refractive index, which leads to the use of perturbation theory. Born perturbation theory is the commonest perturbation theory and is generally applied in research of irradiance scintillations.

Born perturbation theory states that any component of the optical field in the turbulent media can be expressed as the superposition of the free-space solution and perturbation solution of the wave equation:

$$
E=E_{0}+E_{1}+E_{2}+\cdots
$$

where $E_{0}$ is the free-space solution and $E_{1}, E_{2}, \cdots$ are the higher order perturbation solutions resulting from media fluctuation. Neglecting $E_{2}$ and the other higher order items, we find that $E_{1}$ is the sum of a large number of independent spherical waves. According to the central limit theorem, the real and imaginary parts of $E_{1}$ have normal distributions.

We assume that the field strength at a point in the turbulent field is

$$
\begin{aligned}
E=A \exp (i w t) & =(U+i V) \exp (i w t) \\
& =\sqrt{I} \exp ^{i \theta} \exp (i w t),
\end{aligned}
$$

where $A$ is the complex amplitude, $U$ and $V$ are the real and imaginary parts of $A, \theta$ is the phase, $I=A A^{*}$ is the intensity, $w$ is the angular frequency of the assumed monochromatic source, and $i=(-1)^{1 / 2}$. Thus, we have

$$
\begin{aligned}
P(U, V)= & \frac{1}{2 \pi \sigma_{U} \sigma_{V}\left(1-\rho^{2}\right)^{1 / 2}} \\
& \times \exp \left[\frac{-1}{2\left(1-\rho^{2}\right)}\left(\frac{\Delta U^{2}}{\sigma_{U}^{2}}-2 \rho \frac{\Delta U \Delta V}{\sigma_{U} \sigma_{V}}+\frac{\Delta V^{2}}{\sigma_{V}^{2}}\right)\right],
\end{aligned}
$$

where $\sigma_{U}$ and $\sigma_{V}$ are the standard deviations of $U$ and $V$, $\rho=\left\langle\Delta U \Delta V>/ \sigma_{U} \sigma_{V}\right.$ is the correlation coefficient of $U$ and $V$, $\Delta U=U-<U>, \Delta V=V-<V>$, and the angular brackets $<>$ denote ensemble averages.

\subsection{Equiprobability density ellipse}

Since it is difficult to directly obtain a simple analytical solution to eq. (3), we make $\rho=0$ through an appropriate coordinate rotation. Thus, we have

$$
P(X, Y)=\frac{1}{2 \pi \sigma_{X} \sigma_{Y}} \times \exp \left[-\frac{1}{2}\left(\frac{\Delta X^{2}}{\sigma_{X}^{2}}+\frac{\Delta Y^{2}}{\sigma_{Y}^{2}}\right)\right],
$$

where the characteristics of the rotation are graphically illustrated by an equiprobability density ellipse. Here, the equiprobability density ellipse is defined by the line along which the probability density is $1 / \sqrt{e}$ times the maximum value, and shown in Figure 1. The five parameters $\langle U\rangle$,

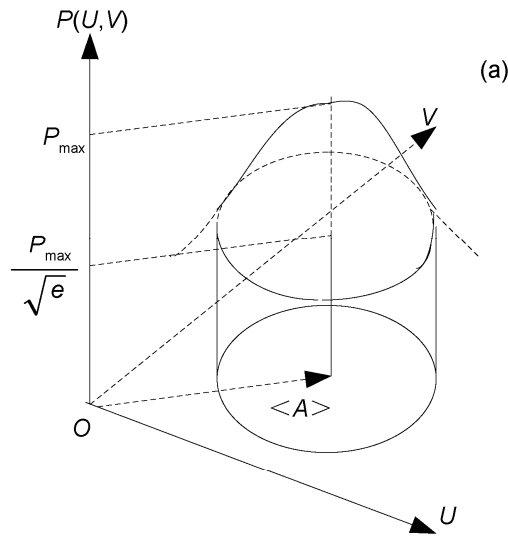

(a)

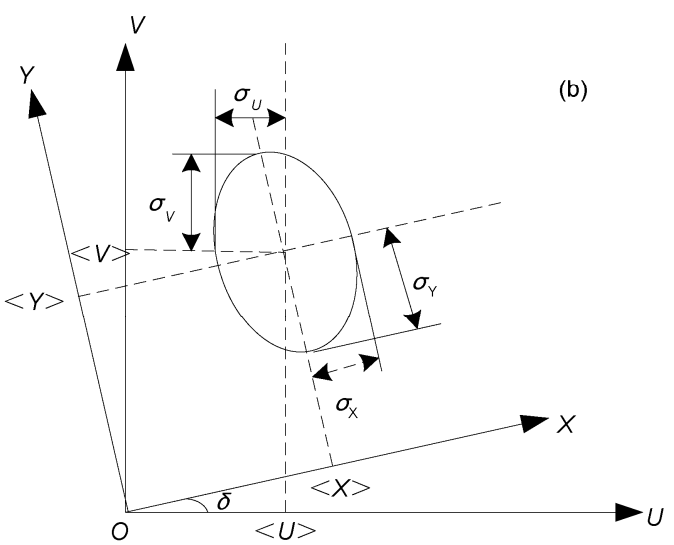

Figure 1 Joint probability density function (a) and equiprobability density ellipse (b).

$<V>, \sigma_{U}, \sigma_{V}$ and $\rho$ in eq. (3) uniquely determine the position, shape and orientation of the equiprobability density ellipse.

The equation for the equiprobability density ellipse in the new $U-V$ coordinate system is [12]

$$
\frac{\Delta U^{2}}{\sigma_{U}^{2}}-\frac{2 \rho \Delta U \Delta V}{\sigma_{U} \sigma_{V}}+\frac{\Delta V^{2}}{\sigma_{V}^{2}}=\left(1-\rho^{2}\right) .
$$

However, the equation for the equiprobability density ellipse in the $X-Y$ coordinate system is

$$
\frac{\Delta X^{2}}{\sigma_{X}^{2}}+\frac{\Delta Y^{2}}{\sigma_{Y}^{2}}=1
$$

where the $X-Y$ coordinates have been rotated around the origin from the $U-V$ coordinate system through the angle

$$
\delta=\frac{1}{2} \tan ^{-1}\left[2 \sigma_{U} \sigma_{V} \rho /\left(\sigma_{U}^{2}-\sigma_{V}^{2}\right)\right] .
$$

It is seen by referring to Figure 1 that the new variables $X$ and $Y$ in eq. (4) satisfy the relations

$$
\Delta X=X-\langle X\rangle, \Delta Y=Y-\langle Y\rangle,
$$




$$
\begin{gathered}
\langle X\rangle=\langle U\rangle \cos \delta+\langle V\rangle \sin \delta, \\
\langle Y\rangle=\langle V\rangle \cos \delta-\langle U\rangle \sin \delta, \\
\sigma_{X}^{2}=\left(\sigma_{U}^{2} \cos ^{2} \delta-\sigma_{V}^{2} \sin ^{2} \delta\right) / \cos 2 \delta, \\
\sigma_{Y}^{2}=\left(\sigma_{V}^{2} \cos ^{2} \delta-\sigma_{U}^{2} \sin ^{2} \delta\right) / \cos 2 \delta .
\end{gathered}
$$

The intensity satisfies

$$
I=A A^{*}=U^{2}+V^{2}=X^{2}+Y^{2}
$$

and the associated phase angle is

$$
\mu=\theta-\delta=\tan ^{-1}(Y / X)
$$

as defined in the $X-Y$ coordinate system.

\section{Probability density functions of the random fluctuation intensity and corresponding phase}

\subsection{Exact models}

Assuming that $\tilde{I}_{X}=(\Delta X)^{2}$ and $\tilde{I}_{Y}=(\Delta Y)^{2}$, we define $\tilde{I}=\Delta A \cdot \Delta A^{*}$ as the random fluctuation intensity, where $\Delta A=A-\langle A\rangle$, $A=X+i Y$ and $\langle A\rangle=\langle X>+i<Y>$; we thus have

$$
\begin{gathered}
\tilde{I}=\Delta X^{2}+\Delta Y^{2}=\tilde{I}_{X}+\tilde{I}_{Y}, \\
\langle\tilde{I}\rangle=\left\langle\tilde{I}_{X}\right\rangle+\left\langle\tilde{I}_{Y}\right\rangle=\sigma_{X}^{2}+\sigma_{Y}^{2} .
\end{gathered}
$$

We assume that $m=\Delta X$ and $n=\Delta Y$ and introduce them into eq. (4) to yield

$$
P(m, n)=\frac{1}{2 \pi \sigma_{X} \sigma_{Y}} \times \exp \left[-\frac{1}{2}\left(\frac{m^{2}}{\sigma_{X}^{2}}+\frac{n^{2}}{\sigma_{Y}^{2}}\right)\right],
$$

where $\tilde{I}=m^{2}+n^{2}$. Changing to polar coordinates, we have

$$
\begin{aligned}
& P(\tilde{I}, \mu) \\
& =\frac{1}{4 \pi \sigma_{X} \sigma_{Y}} \times \exp \left[-\frac{1}{2}\left(\frac{\tilde{I} \cos ^{2} \mu}{\sigma_{X}^{2}}+\frac{\tilde{I} \sin ^{2} \mu}{\sigma_{Y}^{2}}\right)\right],
\end{aligned}
$$

where $\mu=\arctan (n / m)$. Integrating eq. (14) with respect to $\mu$, the marginal PDF of the random fluctuation intensity is obtained as

$$
\begin{aligned}
p(\tilde{I}) & =\int_{0}^{2 \pi} P(\tilde{I}, \mu) \mathrm{d} \mu \\
& =\frac{1}{2 \sigma_{X} \sigma_{Y}} \exp \left[-\frac{\sigma_{X}^{2}+\sigma_{Y}^{2}}{4 \sigma_{X}^{2} \sigma_{Y}^{2}} \tilde{I}\right] I_{0}\left[\frac{\sigma_{X}^{2}-\sigma_{Y}^{2}}{4 \sigma_{X}^{2} \sigma_{Y}^{2}} \tilde{I}\right]
\end{aligned}
$$

where $I_{0}$ is a modified Bessel function of the first kind. This model is called an exponential-Bessel distribution, and is the exact model of the random fluctuation intensity.

Similarly, by integrating eq. (14) with respect to $\tilde{I}$, the marginal PDF of the phase corresponding to the random fluctuation intensity is obtained as

$$
\begin{aligned}
p(\mu) & =\int_{0}^{\infty} P(\tilde{I}, \mu) \mathrm{d} \mu \\
& =\frac{\sigma_{X} \sigma_{Y}}{2 \pi\left(\sigma_{Y}^{2} \cos ^{2} \mu+\sigma_{X}^{2} \sin ^{2} \mu\right)} .
\end{aligned}
$$

From eqs. (14), (15) and (16), we conclude that $P(\tilde{I}, \mu)$ $\neq P(\tilde{I}) \cdot P(\mu)$; that is, the random fluctuation intensity and the corresponding phase are not independent.

\subsection{Circular-Gaussian approximation}

When the accuracy requirement is not so high, the circular-Gaussian approximation is often applied to the PDF; i.e. $\sigma_{X}^{2}=\sigma_{Y}^{2}$. Simplifying eqs. (15) and (16) respectively, the PDFs of the random fluctuation intensity and the corresponding phase are obtained as

$$
\begin{gathered}
p(\tilde{I})=\frac{1}{2 \sigma_{X}^{2}} \exp \left[-\frac{1}{2 \sigma_{X}^{2}} \tilde{I}\right], \\
p(\mu)=\frac{1}{2 \pi} .
\end{gathered}
$$

Therefore, through the circular-Gaussian approximation, the PDFs of the random fluctuation intensity and the phase become a negative-exponential distribution and uniform distribution, respectively. Making the same simplification for eq. (14) yields

$$
\begin{aligned}
& P(\tilde{I}, \mu) \\
& =\frac{1}{4 \pi \sigma_{X}{ }^{2}} \times \exp \left[-\frac{1}{2}\left(\frac{\tilde{I} \cos ^{2} \mu+\tilde{I} \sin ^{2} \mu}{\sigma_{X}^{2}}\right)\right] \\
& =\frac{1}{4 \pi \sigma_{X}{ }^{2}} \times \exp \left[-\frac{1}{2} \frac{1}{\sigma_{X}^{2}} \tilde{I}\right] \\
& =P(\tilde{I}) \cdot P(\mu),
\end{aligned}
$$

which means that when satisfying the circular-Gaussian approximation, the random fluctuation intensity and the corresponding phase will be independent.

\section{Analysis}

Here, we assume $\langle\tilde{I}\rangle=1\left(\sigma_{X}^{2}+\sigma_{Y}^{2}=1\right)$ and introduce a degree of circularity $C$, defined as the ratio of the lengths of the axes of the ellipse in Figure 1:

$$
C=\sigma_{X} / \sigma_{Y} .
$$

Figure 2 presents the PDF of the random fluctuation intensity. It shows the dependence of the exponential-Bessel 


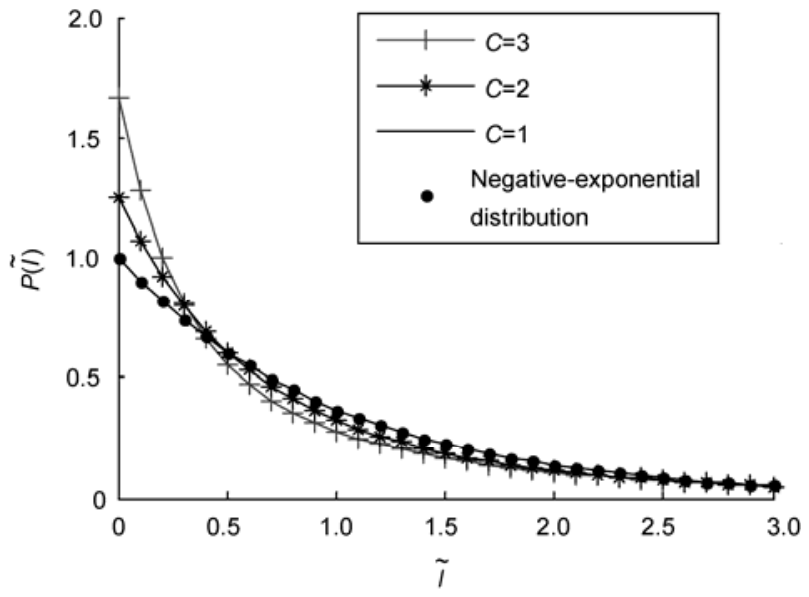

Figure 2 PDF model of the random fluctuation intensity.

distribution of eq. (15) on $C$. With an increase in $C$, the exponential-Bessel distribution line gradually approaches the negative-exponential line until they meet at $C=1$. Therefore, the negative-exponential distribution can be regarded as, but cannot replace, the exponential-Bessel distribution, for it is only an approximate situation, which is consistent with the theoretical conclusion above.

Figure 3 plots the PDF of the corresponding phase, and demonstrates the dependence of the PDF on $C$. We find that the model is symmetric around the center of $\mu=\pi$. That is, the phase is periodic with period $\pi$. With an increase in $C$, the PDF of the phase becomes increasingly smooth until the

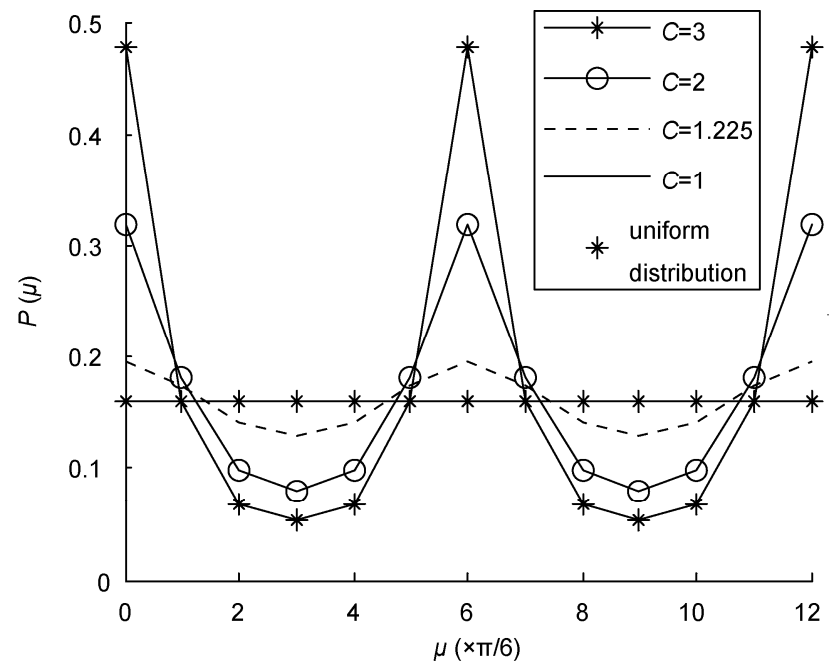

Figure 3 PDF model of the corresponding phase. distribution is uniform, which is consistent with the theoretical conclusion above.

\section{Conclusion}

On the basis of Born perturbation theory, this paper introduces the concept of the random fluctuation intensity and then deduces and analyzes the exact PDFs of this new parameter and its corresponding phase using an equiprobability density ellipse. Finally, we verify the deduced models through simulation and conclude that the random fluctuation intensity and the corresponding phase are independent only under the circular-Gaussian approximation.

The random fluctuation intensity not only reflects the fluctuation of the intensity in the turbulence media, but also has an exact model. Therefore, it would be useful for replacing intensity in some applications.

This work was supported by the Special Foundation Program for Taishan Mountain Scholars.

1 Tatarski V I. Wave Propagation in a Turbulent Medium. New York: McGraw-Hill Book Company, 1961

2 Katsisa A, Nistazakis H E, Tombras G S. Bayesian and frequentist estimation of the performance of free space optical channels under weak turbulence conditions. J Franklin Inst, 2009, 346: 315-327

3 Ivan B D, Goran T D. On the communication over strong atmospheric turbulence channels by adaptive modulation and coding. Opt Express, 2009, 17: 18250-18262

4 Churnside J H, Clifford S F. Log-normal Rician probability-density function of optical scintillations in the turbulent atmosphere. J Opt Soc Am A, 1987, 4: 1923-1930

5 Zhang Y X, Chi Z Y. Transimission and Imaging of Light Waves in the Atmosphere (in Chinese). Beijing: National Defense Industry Press, 1997. 17-22, 110-123

6 Rao R Z. Light Propagation in the Turbulent Atmosphere (in Chinese). Hefei: Anhui Science and Technology Publishing House, 2005. 117-124, 179-199

7 Bissonnette L R, Wizinowich P L. Probability distribution of turbulent irradiance in a saturation regime. Appl Opt, 1979, 18: 1590-1599

8 Fremouw E J, Livingston R C, Miller D A. On the statistics of scintillating signals. J Atmos Terrest Phys, 1980, 42: 717-731

9 Zhang Y X, Chi Z Y. The first order probability density function of the intensity and phase of laser speckle (in Chinese). Chinese Sci Bull (Chinese Ver), 1991, 36: 661-664

10 Zhang Y X, Chi Z Y. Statistical distribution of the integrated intensity of noncircular Gaussian laser speckle. Chin J Lasers, 1989, 18: 230-235

11 Zhang Y X, Chi Z Y. Statistical properties of the intensity and phase for laser propagation through the turbulent atmosphere. Opto-Electr Eng, 1992, 19: 13-19

12 Uozumi J, Asakura T. First-order probability density function of the laser speckle phase. Opt Quant Electr, 1980, 12: 477-494

Open Access This article is distributed under the terms of the Creative Commons Attribution License which permits any use, distribution, and reproduction in any medium, provided the original author(s) and source are credited. 